\title{
Estimation of the rotational terms of the dynamic response matrix
}

\author{
D. Montalvão ${ }^{\mathrm{a}}$, A.M.R. Ribeiro ${ }^{\mathrm{b}}$, N.M.M. Maia ${ }^{\mathrm{b}, *}$ and J.M.M. Silva ${ }^{\mathrm{b}}$ \\ ${ }^{a}$ Department of Mechanical Engineering, Escola Superior de Tecnologia, Polytechnic Institute of Set úbal Campus \\ do IPS, Estefanilha, 2910-761 Setúbal, Portugal \\ ${ }^{\mathrm{b}}$ Department of Mechanical Engineering, Instituto Superior Técnico, Technical University of Lisbon Av. Rovisco \\ Pais, 1049-001 Lisboa, Portugal
}

This paper is a modest tribute to the memory of Bruno Piombo, a very dearest colleague and friend, with whom we spent so many good moments.

\begin{abstract}
The dynamic response of a structure can be described by both its translational and rotational receptances. The latter ones are frequently not considered because of the difficulties in applying a pure moment excitation or in measuring rotations. However, in general, this implies a reduction up to $75 \%$ of the complete model. On the other hand, if a modification includes a rotational inertia, the rotational receptances of the unmodified system are needed. In one method, more commonly found in the literature, a so called T-block is attached to the structure. Then, a force, applied to an arm of the T-block, generates a moment together with a force at the connection point. The T-block also allows for angular displacement measurements. Nevertheless, the results are often not quite satisfactory. In this work, an alternative method based upon coupling techniques is developed, in which rotational receptances are estimated without the need of applying a moment excitation. This is accomplished by introducing a rotational inertia modification when rotating the T-block. The force is then applied in its centroid. Several numerical and experimental examples are discussed so that the methodology can be clearly described. The advantages and limitations are identified within the practical application of the method.
\end{abstract}

Keywords: Rotational degrees of freedom (RDOFs), structural modification, coupling, dynamic response, modal analysis

\section{Introduction}

It is nowadays recognized that rotational degrees of freedom (RDOFs) may represent an important role in the characterization of the dynamic behaviour of real structures. One of the mathematical models used in the description of the dynamical behaviour of a structure is the response model, which relates the response to an excitation at two given locations. The response may either be a linear displacement (translation) or an angular displacement (rotation) and the excitation may either be a force or a moment. Thus, one can consider relations between translation and force, translation and moment, rotation and force and rotation and moment. The measurement equipments used nowadays allow for obtaining the relationships between translation and force with enough accuracy. However, the other relations are often not considered because of the difficulties in exciting the structures with pure moments. It is clear that if rotations and moments are neglected, up to $75 \%$ of the model used to describe the dynamic behaviour of a structure at a given location will not be known.

Various authors have discussed the utility of including RDOFs in dynamic models. In coupling techniques, consideration of rotations in the coupling co-ordinates may be decisive, as they can be responsible for force or

*Corresponding author. Tel.: +3512184 17454; Fax: +3512184 17915; E-mail: nmaia@dem.ist.utl.pt. 
moment transmissions between the various substructures [2,6,32]. On the other hand, in the field of Structural Dynamic Modification (SDM) and Updating [4], consideration of rotations seems to have a strong importance, as data are acquired on a prototype to validate and correct a Finite Element Model (FEM), which, once verified, can be used to evaluate project alterations. Also, if a modification is to include a rotational inertia or rotational stiffness, the rotational receptances of the unmodified system are needed $[9,18]$. Furthermore, the combined use of translations and rotations may reduce the number of measurements that are necessary to represent the vibration modes with an accuracy identical to the one which would be obtained by measuring only translations [16].

The following is mainly based on the very comprehensive surveys given in references [13] and [18].

When talking about RDOFs, there are two problems to be considered: the measurement and the excitation.

The first has already been approached by several authors, showing that measurements of angular displacements can be done with little uncertainty. The first solution is the use of appropriate response transducers, which have the disadvantage of being much more expensive than conventional response transducers. In one method, more commonly found in the literature [2,5-7,9,13,20,25,28,31], a so called T-block is attached to the structure, allowing for the measurement of translations at two locations conveniently chosen which are converted into rotations by the use of simple geometrical relations. In a different technique, Cafeo et al. [16] measure a translation and two rotations simultaneously with no physical contact. Bokelberg et al. [10,11] developed a multidirectional transducer, which allows for the measurement of the six DOFs in a single location using three LASER vibrometers pointed on a tetrahedrical device placed on the structure. Nevertheless, the device dimensions are still considerable. Another multidirectional response transducer, consisting of six piezoelectric accelerometers conveniently displaced in a triangular geometry, was developed within the scope of a EU contract Brite Euram PR-CT97-0540 [13]. Other alternative solutions to the measurement of rotations can be found in that work, such as the use of a LASER vibrometer, which avoids physical contact to the structure and allows for a scanning process by moving the beam from one measurement site to the next in a controlled way [8]. Stanbridge and Ewins use a similar approach in [1].

Considering the problem of exciting the structure with a moment and measuring it, many works have been carried out in the pursuit of an effective solution. Still, a generally accepted technique has not yet been developed, as a wide variety of limitations is usually present. Application of a single point pure moment is, for practical reasons, very difficult to accomplish. Based on the definition of binary, Smith [12] used two shakers in such a configuration that it would be possible to excite the structure with two identical forces with opposite directions. Thirty years later, Ribeiro [2] used a similar approach, concluding that the main problems of this method are related to differences in the impedances between the two shakers and to the structure reactions, giving no guarantee that the applied forces are, in every moment, perfectly symmetrical. Sanderson and Fredo [23] and Sanderson [24] used two rigid blocks, in the shape of a T-block and an I-block, showing that the errors arise from the moment applied to the structure and from the uncertainties on the measurement of the applied moment. The mass of the moment exciter is also said to be a major obstacle. In the already mentioned EU contract Brite Euram PR-CT97-0540 [13], a low weight pure moment exciter is described. Trethewey and Sommer [26] presented a device which allows for generating a pure moment by the centrifugal forces generated by eccentric masses symmetrically attached to two rotating wheels. Again, the weight of the device came out to be a major drawback. Furthermore, several other restrictions may be found, such as the narrow frequency range, limitation to harmonic excitations and problems related to the fixture.

The method studied by Ewins and Sainsbury [5], Ewins and Gleeson [6], Sainsbury [25], Silva [20] and Ewins and Silva [7] is one of the first attempts to measure rotational receptances. Using a rigid T-shaped block, they showed that the full matrix of receptances can be derived from the knowledge of the measured translational receptances. However, results are often not accurate enough, namely when using them for substructural coupling. Mottershead et al. [17] have recently proposed a technique in which a T-block is treated as a modification at the point of its attachment deriving the full forcing and response vector at the connection point. The FEM of the T-block has been used in order to include its stiffness in the formulation, thus avoiding ill-conditioning problems.

Maia et al. [21,30] proposed a method, based on impedance coupling techniques, in which rotational receptances are estimated without having to measure them. It is also shown that neither a moment exciter nor an eccentric force applied to a rigid fixture are needed in the estimation of rotational receptances. This method will be exploited throughout this paper, though following a different approach, where uncoupling is undertaken in steps and not simultaneously. The method developed herein is also based on the one proposed by Maia et al. [22,29] for the estimation of the full matrix of receptance of an MDOF translation system from a single column or line of the receptance matrix corresponding to an applied force. 


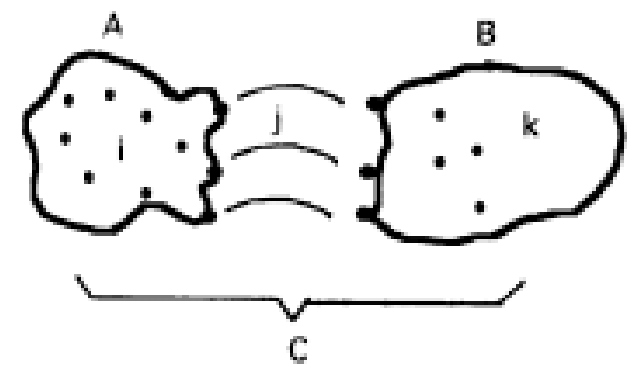

Fig. 1. Structure C, composed by two substructures A and B, connected together. Notation for the sets of co-ordinates used.

The main goal of this work is to provide a new approach for the determination of a rotational response to a moment excitation without application (and measurement) of a moment, exploiting its limitations and studying in a comprehensive way the main reasons that may be in the origin of poor results.

\section{Theoretical development}

\subsection{Basic equations}

The method developed herein is based in the Mechanical Impedance Coupling Technique. Consider two separate structures, called $A$ and $B$. When rigidly connected through some co-ordinates, a new structure, called $C$, is constituted. One may represent the domains of substructures $A$ and $B$, coupled by a finite number of coordinates, so that, together, they form structure $C$. Naming the coordinates that belong exclusively to $A$ by $i$, those which belong exclusively to $B$ by $k$ and those which are common to both by $j$, i.e., the connection ones, the respective domains may be represented as shown schematically in Fig. 1.

Let also: $\left[H_{i i}^{(A)}\right]$ represent the matrix of accelerance FRFs amongst co-ordinates $i ;\left[H_{i j}^{(A)}\right]$ and $\left[H_{j i}^{(A)}\right]$ represent the matrix of accelerance FRFs amongst co-ordinates $i$ and $j ;\left[H_{j j}^{(A)}\right]$ represent the matrix of accelerance FRFs amongst co-ordinates $j$ belonging to substructure $A ;\left[H_{k k}^{(B)}\right]$ represent the matrix of accelerance FRFs amongst co-ordinates $k ;\left[H_{k i}^{(B)}\right]$ and $\left[H_{j k}^{(B)}\right]$ represent the matrix of accelerance FRFs amongst co-ordinates $k$ and $j ;\left[H_{j j}^{(B)}\right]$ represent the matrix of accelerance FRFs amongst co-ordinates $j$ belonging to substructure $B$. Hence, the matrix of accelerance FRFs of substructures $A$ and $B$ and of the resulting structure $C$ may be given by the following expressions:

$$
\begin{aligned}
& {\left[H^{(A)}\right]=\left[\begin{array}{l}
{\left[H_{i i}^{(A)}\right]\left[H_{i j}^{(A)}\right]} \\
{\left[H_{j i}^{(A)}\right]\left[H_{j j}^{(A)}\right]}
\end{array}\right]} \\
& {\left[H^{(B)}\right]=\left[\begin{array}{l}
{\left[H_{j j}^{(B)}\right]\left[H_{j k}^{(B)}\right]} \\
{\left[H_{k j}^{(B)}\right]\left[H_{k k}^{(B)}\right]}
\end{array}\right]} \\
& {\left[H^{(C)}\right]=\left[\begin{array}{l}
{\left[H_{i i}^{(C)}\right]\left[H_{i j}^{(C)}\right]\left[H_{i k}^{(C)}\right]} \\
{\left[H_{j i}^{(C)}\right]\left[H_{j j}^{(C)}\right]\left[H_{j k}^{(C)}\right]} \\
{\left[H_{k i}^{(C)}\right]\left[H_{k j}^{(C)}\right]\left[H_{k k}^{(C)}\right]}
\end{array}\right]}
\end{aligned}
$$

Applying the appropriate equilibrium and compatibility conditions at the common co-ordinates (constituting the joining locations), one may conclude that the coupling of both substructures will result in a system $C$ with an accelerance matrix that may be written as a function of both matrixes of accelerance of substructures $A$ and $B$ [28]:

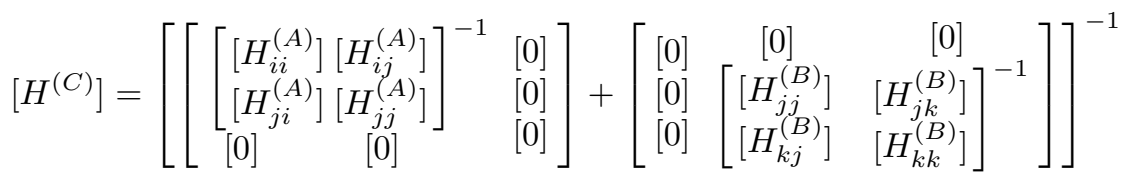




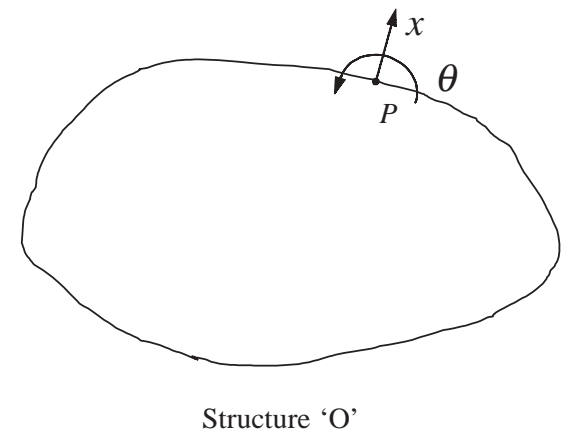

Fig. 2. Co-ordinates $x$ and $\theta$ at location $P$ of structure 'O'.

Because this formulation requires three matrix inversions, thus becoming computationally "heavy", one may write an alternative form of Eq. (4) as proposed in [2,22]:

$$
\left.\left[H^{(C)}\right]=\left[\begin{array}{ccc}
{\left[H_{i i}^{(A)}\right]} & {\left[H_{i j}^{(A)}\right]} & {[0]} \\
{[0]} & {[0]} & {\left[H_{j k}^{(B)}\right]} \\
{[0]} & {[0]} & {\left[H_{k k}^{(B)}\right]}
\end{array}\right]+\left[\begin{array}{c}
-\left[H_{i j}^{(A)}\right] \\
{\left[H_{j j}^{(B)}\right]} \\
{\left[H_{k j}^{(B)}\right]}
\end{array}\right] \cdot\left(\left[H_{j j}^{(A)}\right]+\left[H_{j j}^{(B)}\right]\right)^{-1} \cdot\left[\begin{array}{c}
{\left[H_{j i}^{(A)}\right]} \\
{\left[H_{j j}^{(A)}\right]} \\
-\left[H_{j k}^{(B)}\right]
\end{array}\right]\right]^{T}
$$

where the matrix to invert is of the order of the number of co-ordinates common to $A$ and $B$.

\subsection{Estimation of rdofs}

Normally, a T-block is used to perform two distinct functions: to allow for an accurate measurement of the tangent to the deformation of the structure during vibration (by use of finite differences) and to allow for a moment excitation, together with a force, at the coupling point between the T-block and the structure (by applying a single force through an arm of the T-block). In this work, the excitation force will be applied through the centroid of the T-block and we will assume that the tangent and the secant to the structural deformation are parallel, so that one can make measurements either on the arms of the T-block or directly on the structure. The novelty of this method is to explore a third function that the T-block may perform: to introduce a rotational inertia modification by rotating the T-block around one of its symmetry axis.

The best way to explain how the proposed technique works is to use an example where measurements are made in two co-ordinates. Hence, consider a structure, named ' $\mathrm{O}$ ', with two co-ordinates of interest $x$ and $\theta$ at location $P$ (Fig. 2).

Suppose the intention is to characterize its dynamic behaviour, relating the two co-ordinates of interest by measuring the corresponding FRFs. The structure will be excited with a shaker at co-ordinate and the force and acceleration signals will be measured through a force transducer and response transducers respectively. Using a finite difference procedure, it is clear that one can easily access $H_{x x}, H_{x \theta}$ and, considering reciprocity, $H_{\theta x}$. The problem is that, to measure $H_{\theta \theta}$, it seems that one needs to apply a moment through co-ordinate $\theta$. In fact, that is not necessary, as will be shown.

Coupling a T-block at point $P$, one obtains structure ' $T_{1}$ ' (Fig. 3(a)), which, by rotating $90^{\circ}$ around its vertical axis, is turned into structure ' $T_{2}$ ' (Fig. 3(b)). For purposes of this procedure explanation, the mass effects of transducers and T-block will be neglected.

Noting that the contact base of the T-block is a square, the main difference between structure ' $T_{1}$ ' and ' $T_{2}$ ' is a rotational inertia with a value of $I_{2}-I_{1}$ (thus, only small changes in the local stiffness are expected, which will be neglected). Estimation of the rotational FRF $H_{\theta \theta}$ will be accomplished by cancellation of the rotational inertia $I_{2}-I_{1}$.

The measurement technique is very simple. Using conventional response and force transducers, one can measure, for each structure, the force (which is applied at co-ordinate $x$ ) and translational responses at coordinates $x_{A}$ and $x_{B}$, so that FRFs $H_{x x_{A}}$ and $H_{x x_{B}}$ can be known. To obtain the FRFs $H_{x x}$ and $H_{x \theta}$ the expressions are: 


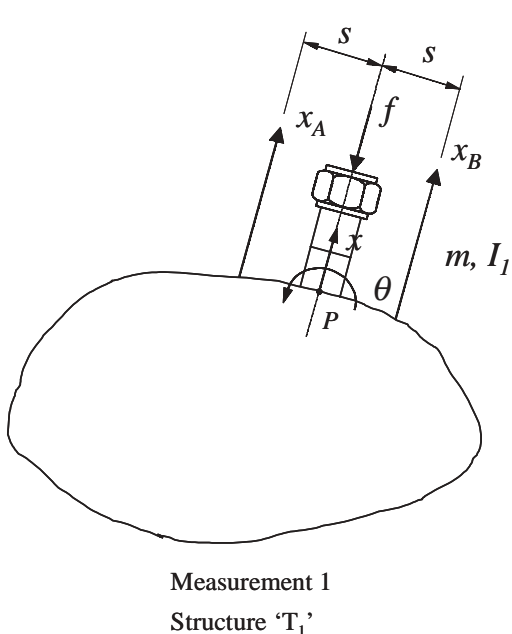

a)

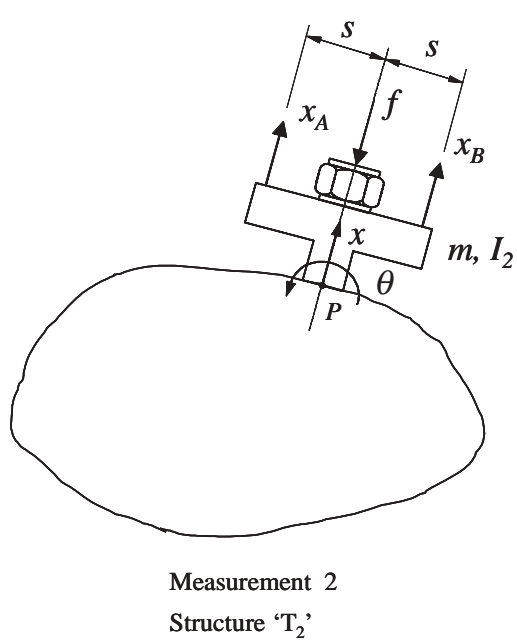

b)

Fig. 3. Coupling a T-block at point $P$ of structure ' $\mathrm{O}$ ', one obtains structure ' $T$ ' (a), which, by rotating $90^{\circ}$ around its vertical axis, is turned into structure ' $T_{2}$ ' (b).

$$
\begin{aligned}
& H_{x x}=\frac{H_{x x_{A}}+H_{x x_{B}}}{2} \\
& H_{x \theta}=\frac{H_{x x_{B}}-H_{x x_{A}}}{2 s}
\end{aligned}
$$

As a result, one should have two pairs of FRFs: $H_{x \theta}^{\left(T_{1}\right)}$ with $H_{x \theta}^{\left(T_{1}\right)}$ and $H_{x \theta}^{\left(T_{2}\right)}$ with $H_{x \theta}^{T_{2}}$.

The structure ' $T_{2}$ ' represented in Fig. 3(b) can be seen as the result of coupling ' $T_{1}$ ' to $I=I_{2}-I_{1}$ and therefore, according to the notation used previously in Section 2.1, it may be stated that:

$$
A \equiv T_{1}, \quad B \equiv I, C \equiv T_{2} \equiv T_{1} \oplus I
$$

where $\oplus$ means "coupled to". As two co-ordinates are being considered, the FRF matrix $\left[H^{(A)}\right]$ will be of order two and $\left[H^{(B)}\right]$ of order one, as it represents a simple rotational inertia modification. Thus, co-ordinate $x$ corresponds now to co-ordinate $i$, co-ordinate $\theta$ corresponds to co-ordinate $j$ and, in this case, there is no co-ordinate $k$. Noting that $H_{\theta \theta}^{(B)}=1 / I$ is the only element in the FRF matrix $\left[H^{(B)}\right]$, and using the same notation as in Fig. 3, Eq. (5) is then reduced to:

$$
\left[\begin{array}{ll}
H_{x x}^{\left(T_{2}\right)} & H_{x \theta}^{\left(T_{2}\right)} \\
H_{\theta x}^{\left(T_{2}\right)} & H_{\theta \theta}^{\left(T_{2}\right)}
\end{array}\right]=\left[\begin{array}{cc}
H_{x x}^{\left(T_{1}\right)} & H_{x \theta}^{\left(T_{1}\right)} \\
0 & 0
\end{array}\right]+\left\{\begin{array}{c}
-H_{x \theta}^{\left(T_{1}\right)} \\
1 / I
\end{array}\right\} \cdot \frac{1}{H_{\theta \theta}^{\left(T_{1}\right)}+1 / I} \cdot\left\{\begin{array}{l}
H_{\theta x}^{\left(T_{1}\right)} \\
H_{\theta \theta}^{\left(T_{1}\right)}
\end{array}\right\}^{T}
$$

which may be written as

$$
\left[\begin{array}{ll}
H_{x x}^{\left(T_{2}\right)} & H_{x \theta}^{\left(T_{2}\right)} \\
H_{\theta x}^{\left(T_{2}\right)} & H_{\theta \theta}^{\left(T_{2}\right)}
\end{array}\right]=\left[\begin{array}{cc}
H_{x x}^{\left(T_{1}\right)}-\frac{H_{\theta x}^{\left(T_{1}\right)} H_{x \theta}^{\left(T_{1}\right)} I}{H_{\theta \theta}^{\left(T_{1}\right)} I+1} H_{x \theta}^{\left(T_{1}\right)}-\frac{H_{x \theta}^{\left(T_{1}\right)} H_{\theta \theta}^{\left(T_{1}\right)} I}{H_{\theta \theta}^{\left(T_{1}\right)} I+1} \\
\frac{H_{\theta x}^{\left(T_{1}\right)}}{H_{\theta \theta}^{\left(T_{1}\right)} I+1} & \frac{H_{\theta \theta}^{\left(T_{1}\right)}}{H_{\theta \theta}^{\left(T_{1}\right)} I+1}
\end{array}\right]
$$

The latter formulation allows for a clearer visualization of the interrelationship between the sub-structures FRFs. Furthermore, solving for the known FRF $H_{\theta x}^{\left(T_{2}\right)}$ (notice again that considering reciprocity $H_{\theta x}=H_{x \theta}$ ):

$$
H_{\theta x}^{\left(T_{2}\right)}=\frac{H_{\theta x}^{\left(T_{1}\right)}}{H_{\theta \theta}^{\left(T_{1}\right)} I+1} \Rightarrow H_{\theta \theta}^{\left(T_{1}\right)}=\frac{1}{I}\left(\frac{H_{\theta x}^{\left(T_{1}\right)}}{H_{\theta x}^{\left(T_{2}\right)}}-1\right)
$$

Finally, solving expression (9) for the unknown FRF $H_{\theta \theta}^{\left(T_{2}\right)}$ : 

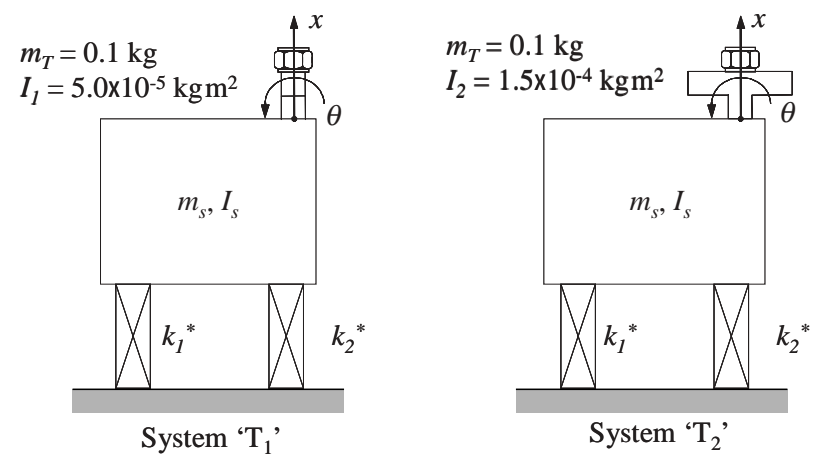

$$
\begin{aligned}
& k_{1}=10000 \mathrm{~N} / \mathrm{m} \quad \eta_{1}=0.005 \\
& k_{2}=7000 \mathrm{~N} / \mathrm{m} \quad \eta_{2}=0.015 \\
& k_{1}^{*}=k_{1}\left(1+\mathrm{i} \eta_{1}\right) \\
& k_{2}{ }^{*}=k_{2}\left(1+\mathrm{i} \eta_{2}\right) \\
& m_{s}=0.5 \mathrm{~kg} \\
& I_{s}=5 \times 10^{-4} \mathrm{kgm}^{2} \\
& 2 s=0.02 \mathrm{~m}
\end{aligned}
$$

Fig. 4. Two DOF numerical example with hysteretic damping.

$$
H_{\theta \theta}^{\left(T_{2}\right)}=\frac{H_{\theta \theta}^{\left(T_{1}\right)}}{H_{\theta \theta}^{\left(T_{1}\right)} I+1} \Rightarrow H_{\theta \theta}^{\left(T_{2}\right)}=\left(\frac{1}{H_{\theta \theta}^{\left(T_{1}\right)}}+I\right)^{-1}
$$

As already said before, it is important to notice that, since $T_{2} \equiv T_{1} \oplus I$, expression (9) states that the FRF matrix of structure ' $T_{2}$ ' is equal to the FRF matrix of structure ' $T_{1}$ ' coupled to the rotational inertia $I=I_{2}-I_{1}$. Hence, the rotational FRF $H_{\theta \theta}^{\left(T_{1}\right)}$ in expression (10) is obtained by cancellation of the rotational inertia $I=I_{2}-I_{1}$, which corresponds to the only difference (at least theoretically) between structures ' $T_{2}$ ' and ' $T_{1}$ '. Once $H_{\theta \theta}^{\left(T_{1}\right)}$ is known, cancellation of additional masses and of the residual rotational inertia $I_{1}$ must be made in order to obtain the FRF of system ' $\mathrm{O}$ ' shown in Fig. 2. The procedure is identical to the one explained above starting with Eq. (5). For purposes of this article that will not be done. Montalvão [9] showed that the main problems that arise with the use of this methodology are directly related with the use of expression Eq. (10).

\section{Examples of application}

\subsection{Numerical example}

In order to analyse the performance of the proposed technique, a very simple numerical example will be used: a two DOF system, with hysteretic damping, as shown in Fig. 4.

Considering that measurements were made as explained in Section 3.2 (Fig. 3), one obtains a set of two pairs of FRFs, each pair corresponding to its respective system or structure: $H_{x \theta}^{\left(T_{1}\right)}$ and $H_{x \theta}^{\left(T_{1}\right)}$ for system ' $T_{1}$ ' and $H_{x \theta}^{\left(T_{2}\right)}$ and $H_{x \theta}^{\left(T_{2}\right)}$ for system ' $T_{2}$ '. Use of Eqs (10) and (11) allow us for the determination of $H_{\theta \theta}^{\left(T_{1}\right)}$ and $H_{\theta \theta}^{\left(T_{2}\right)}$ respectively. Figures 5 and 6 summarize the procedure presenting the FRF curves superimposed so that the performance of the method can be observed.

Despite this extremely good performance, it cannot be forgotten that the method can only be of interest if its application to real data also performs well. It was therefore decided to repeat the previous performance check using the same data and polluting them with random errors. The use of polluted data is meant to simulate real experimental results. Figures 7 and 8 show the results after applying a $\pm 2 \%$ random error to the real and imaginary parts of the "measured" accelerance data $\left(H_{x x_{A}}^{\left(T_{1}\right)}, H_{x x_{B}}^{\left(T_{1}\right)}, H_{x x_{A}}^{\left(T_{2}\right)}\right.$ and $\left.H_{x x_{B}}^{\left(T_{2}\right)}\right)$.

Although $H_{x \theta}^{\left(T_{1}\right)}, H_{x \theta}^{\left(T_{1}\right)}, H_{x \theta}^{\left(T_{2}\right)}$ and $H_{x \theta}^{\left(T_{2}\right)}$ are quite acceptable with the introduction of a random error of $\pm 2 \%$ on the original "measured" data, the same cannot be said for both the derived FRFs $H_{\theta \theta}^{\left(T_{1}\right)}$ and $H_{\theta \theta}^{\left(T_{2}\right)}$. It is clear that the cancellation procedure is very sensitive to errors. Furthermore, it can be seen that, in this particular case, only the region around the first resonance frequency is acceptable. As both the cancellation exercise and the objective of deriving a point FRF that had not been measured previously produce very disappointing results, which shows a strong dependency on measurement errors, and thus, on real data, the use of either modal identification or structural modification techniques (e.g., to incorporate a stiff spring connecting a specific co-ordinate to ground) are suggested in [22]. The former suggestion will be followed when discussing the experimental example in chapter 4. 


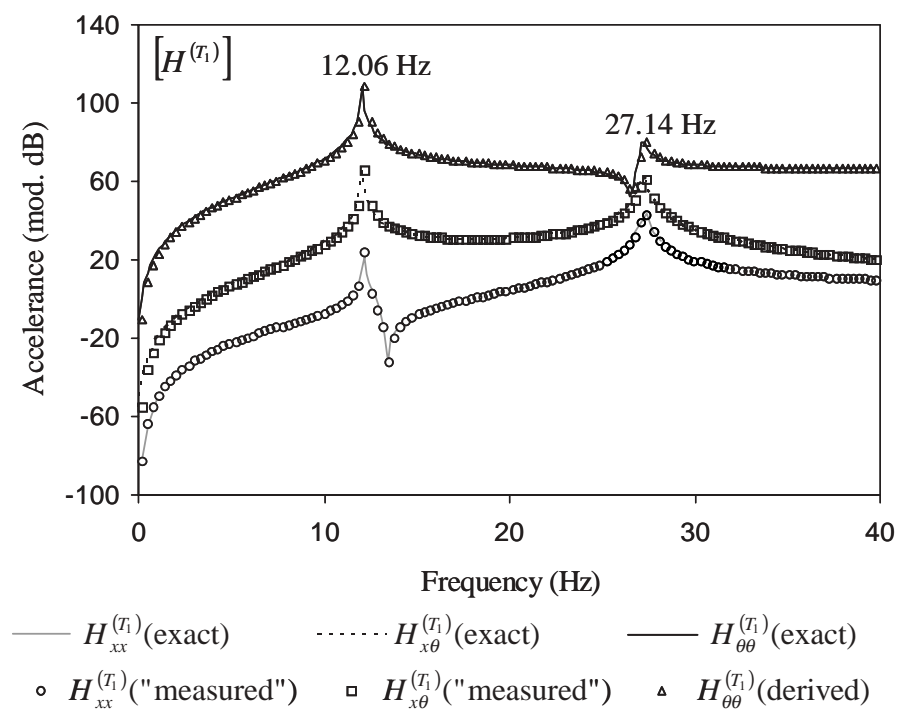

Fig. 5. Theoretical and Simulated terms of the accelerance matrix for numerical system ' $T_{1}$ ' considering two co-ordinates $x$ and $\theta$.

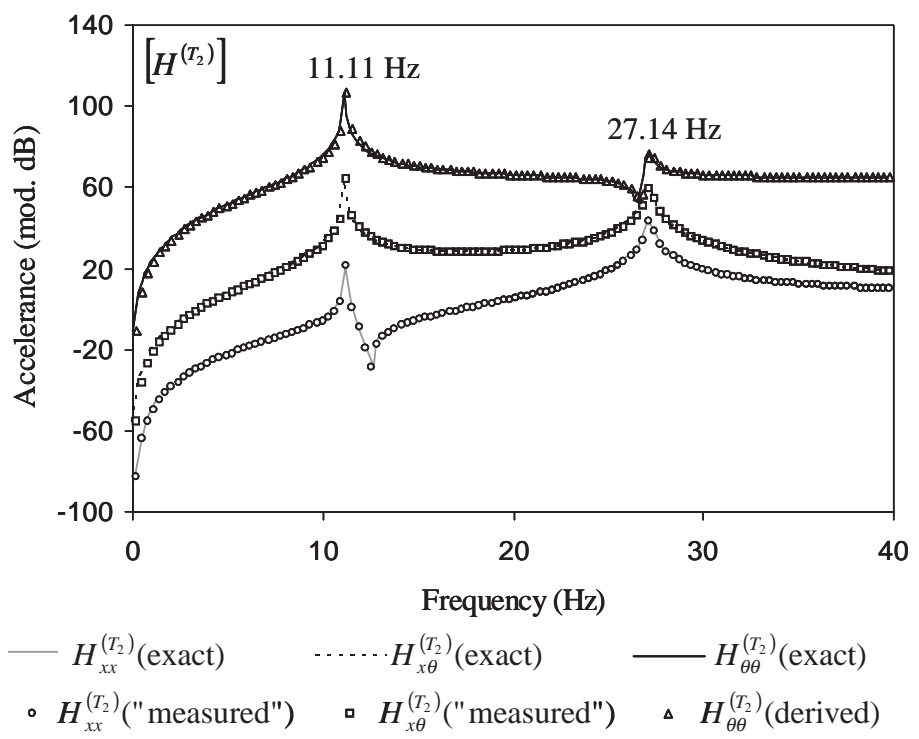

Fig. 6. Theoretical and Simulated terms of the accelerance matrix for numerical system ' $T_{2}$ ' considering two co-ordinates $x$ and $\theta$.

As stated before, when observing the derived FRFs $H_{\theta \theta}^{\left(T_{1}\right)}$ and $H_{\theta \theta}^{\left(T_{2}\right)}$, the region with a better behaviour is around the first resonance frequency. With respect to $H_{x \theta}^{\left(T_{1}\right)}$ and $H_{x \theta}^{\left(T_{2}\right)}$, this is also true, though the error propagation seems to be of less importance. First, notice that the first resonance frequency corresponds to a rotational mode and that the second resonance frequency corresponds to a translational mode (this can be seen in Figs 5-8 by the resonance frequencies shifts caused after cancellation of the rotational inertia). Now consider expressions (7) and (10) used to derive FRFs $H_{x \theta}^{\left(T_{1}\right)}, H_{x \theta}^{\left(T_{2}\right)}$ and $H_{\theta \theta}^{\left(T_{1}\right)}$. The graphical representation of $H_{x \theta}^{\left(T_{1}\right)}, H_{\theta \theta}^{\left(T_{1}\right)}$ and the FRFs involved in their derivation are sufficient for the elaboration of some important remarks (Figs 9 and 10).

Observation of Fig. 9 shows that $\left[H_{x \theta}^{\left(T_{1}\right)}\right]$ seems to be rather noisy above $25 \mathrm{~Hz}$, which is precisely when $\left[H_{x x_{A}}^{\left(T_{1}\right)}\right]$ and $\left[H_{x x_{B}}^{\left(T_{1}\right)}\right]$ have closer amplitude values. This can be easily explained noting that the numerator of Eq. (7) is the subtraction between two similar values. In fact, the difference between two quantities that are similar in value may 


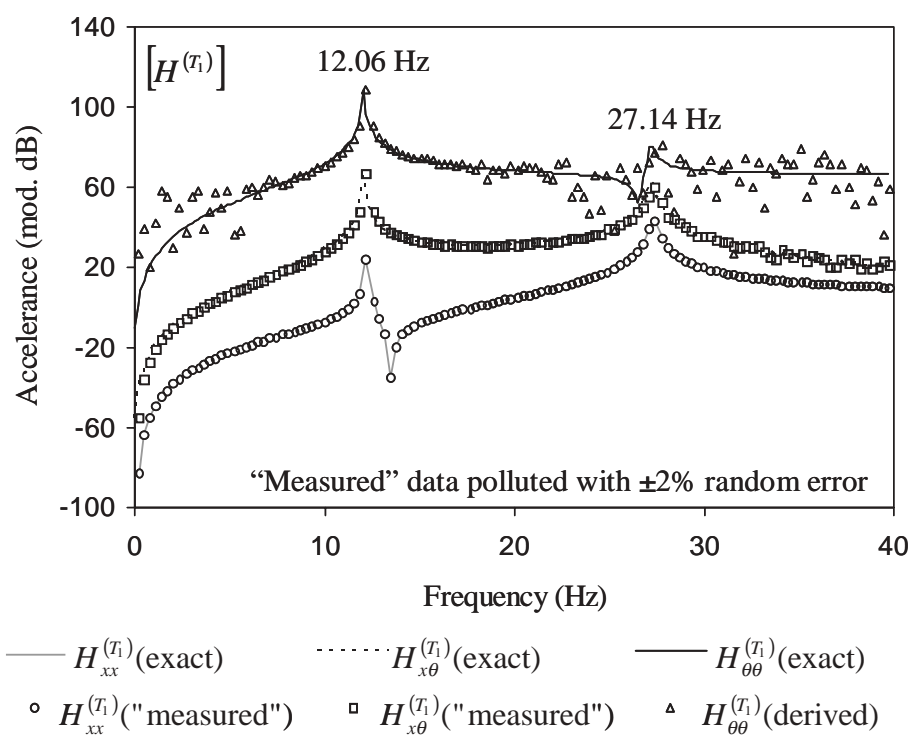

Fig. 7. Theoretical and Simulated (real and imaginary parts of the "measured" data polluted with $\pm 2 \%$ random error) terms of the accelerance matrix for numerical system ' $T$ ' considering two co-ordinates $x$ and $\theta$.

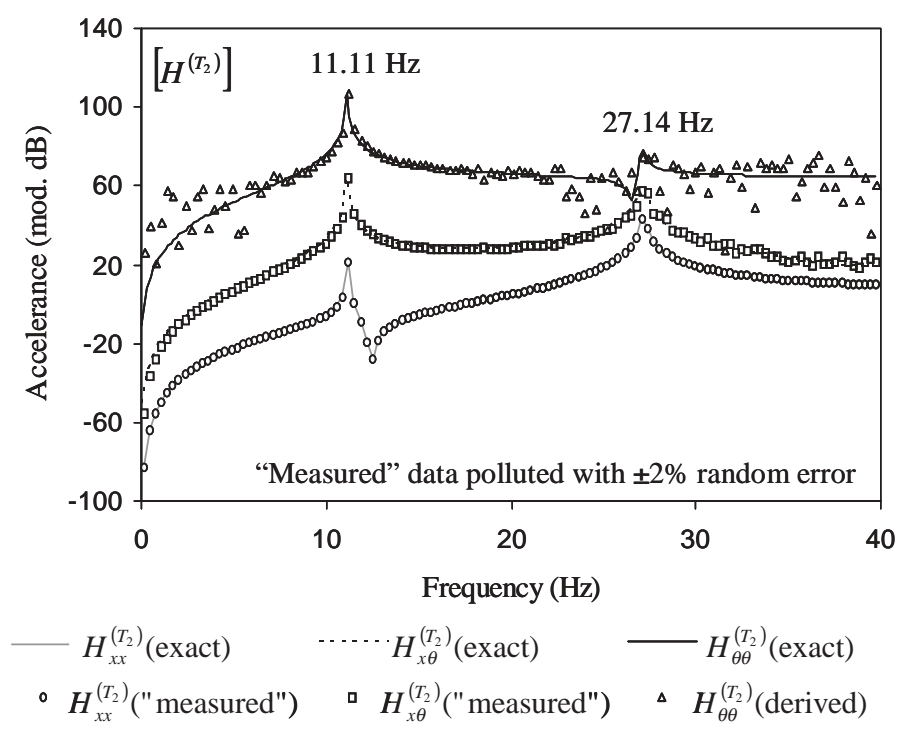

Fig. 8. Theoretical and Simulated (real and imaginary parts of the "measured" data polluted with $\pm 2 \%$ random error) terms of the accelerance matrix for numerical system ' $T 2$ ' considering two co-ordinates $x$ and $\theta$.

be of the same order or smaller than the data errors. This phenomenon is known as subtractive cancellation [15]. Despite a mathematical explanation has been given, a physical explanation can be found too. Once the second resonance frequency is mostly associated to a translation mode, there is little rotation at the measurement point for that frequency, which means that measurements are being made near a peek of vibration.

Figure 10 is very clear with respect to the error propagation in $H_{\theta \theta}^{\left(T_{1}\right)}$. Exception made to the neighbourhood around the first resonance frequency, $H_{\theta \theta}^{\left(T_{1}\right)}$ has no meaning. These are also intervals in which FRFs $H_{x \theta}^{\left(T_{1}\right)}$ and $H_{x \theta}^{\left(T_{2}\right)}$ are very similar. Once again, we are in the presence of a subtractive cancellation numerical problem, as in Eq. (10) one has a subtraction between a quotient near unity (when $H_{x \theta}^{\left(T_{1}\right)}$ has identical values to $H_{x \theta}^{\left(T_{2}\right)}$ ) and unity 


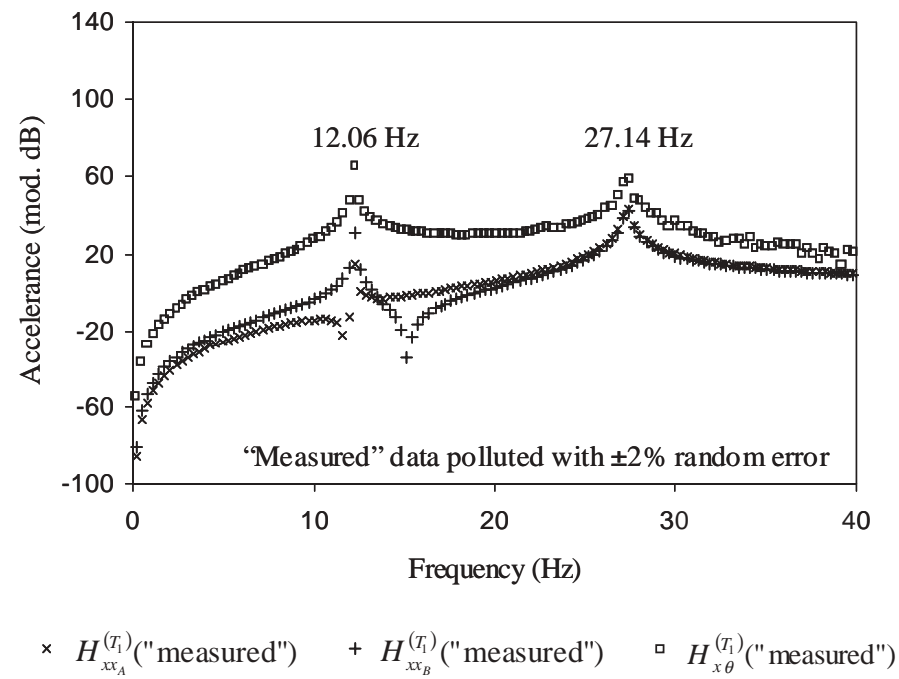

Fig. 9. FRFs involved in expression Eq. (7) (real and imaginary parts of the "measured" data polluted with $\pm 2 \%$ random error).

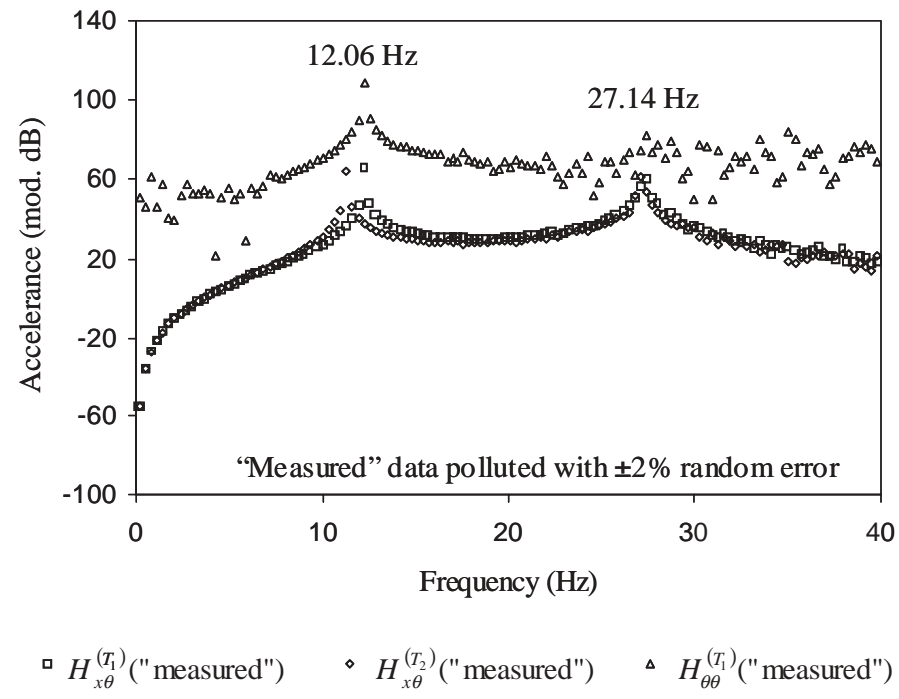

Fig. 10. FRFs involved in expression Eq. (11) (real and imaginary parts of the "measured" data polluted with $\pm 2 \%$ random error).

itself, thus augmenting the introduced $\pm 2 \%$ error. The problem may be said to be ill-conditioned as small changes in data generate big changes in results.

Some explanations were found to justify the type of errors that may appear with real data. The main purpose of this analysis was not to try to find some corrective solutions for these kind of errors (which have already been made in [22]), but to try to understand the problems associated with the method itself and to predict its consequences when applied to real data. Another type of errors, such as those related to the measurement of rotational inertias, have already been approached in [9], but it was concluded that they had little influence on the results.

\subsection{Experimental example}

A very simple experimental set-up was conduced in order to apply the developed method to a practical and real situation. The test structure is a simulated free-free steel beam, with rectangular cross section, with dimensions 

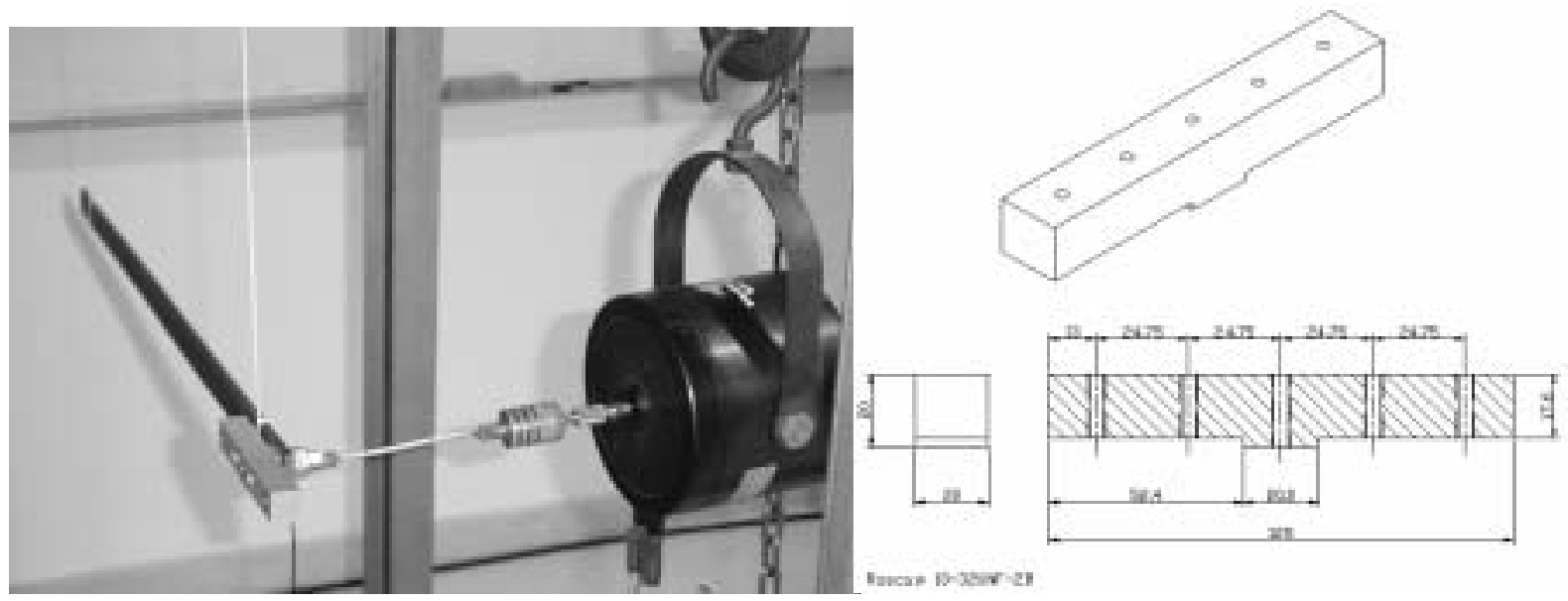

Fig. 11. General experimental setup and T-block dimensions.

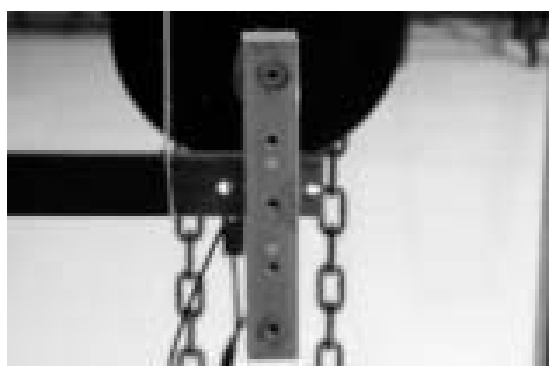

System ' $\mathrm{T}_{1}$ '

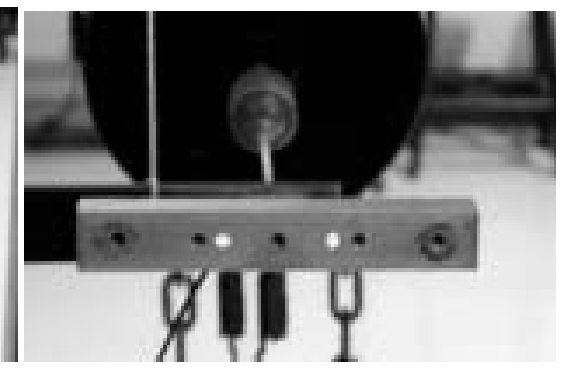

System ' $\mathrm{T}_{2}$ '

Fig. 12. Systems ' $T_{1}$ ' and ' $T_{2}$ ' obtained by rotation of the T-block around one of its symmetry axis.

$800.5 \mathrm{~mm} \times 25.4 \mathrm{~mm} \times 6.3 \mathrm{~mm}$. An aluminium t-shaped block is attached at $20 \mathrm{~mm}$ apart from one of its tips, in order to obtain structures ' $T_{1}$ ' and ' $T_{2}$ ' (Figs 11 and 12).

Measurements were made using a two channel LASER vibrometer and a conventional piezoelectric force transducer, thus yielding a set of two pairs of FRFs: $H_{x x}^{\left(T_{1}\right)}$ and $H_{x \theta}^{\left(T_{1}\right)}$ for system ' $T_{1}$ ' and $H_{x x}^{\left(T_{2}\right)}$ and $H_{x \theta}^{\left(T_{2}\right)}$ for system ' $T_{2}$ '. Finally, $H_{\theta \theta}^{\left(T_{1}\right)}$ and $H_{\theta \theta}^{\left(T_{2}\right)}$ are determined by use of Eqs (10) and (11) respectively. Figures 12 and 13 summarize the procedure presenting the experimental FRF curves and the theoretical ones (obtained by Timoshenko beam theory $[14,19])$ superimposed so that the performance of the method can be observed.

\section{Discussion}

As it has already been observed in the numerical method, the terms $H_{\theta \theta}^{\left(T_{1}\right)}$ and $H_{\theta \theta}^{\left(T_{2}\right)}$ are rather noisy, when compared to the terms used for their derivation, $H_{x \theta}^{\left(T_{1}\right)}$ and $H_{x \theta}^{\left(T_{2}\right)}$. One solution that is pointed out to minimize the measurement errors propagation and their amplification, and thus to obtain cleaner FRF elements, is based on the regeneration of the curves by mean of identification of the modal parameters.

Curves $H_{x \theta}^{\left(T_{1}\right)}, H_{x \theta}^{\left(T_{1}\right)}, H_{x \theta}^{\left(T_{2}\right)}$ and $H_{x \theta}^{\left(T_{2}\right)}$ were identified according to the modal identification technique which uses the so-called Characteristic Response Function (CRF) [27]. Once the modal parameters are identified, it is possible to write the FRF function, which in the case of a receptance is:

$$
\alpha_{j k}(\omega)=\frac{\bar{X}_{j}}{F_{k}}=\sum_{r=1}^{N} \frac{{ }_{r} \bar{A}_{j k}}{\omega_{r}^{2}-\omega^{2}+i \eta_{r} \omega_{r}^{2}}
$$




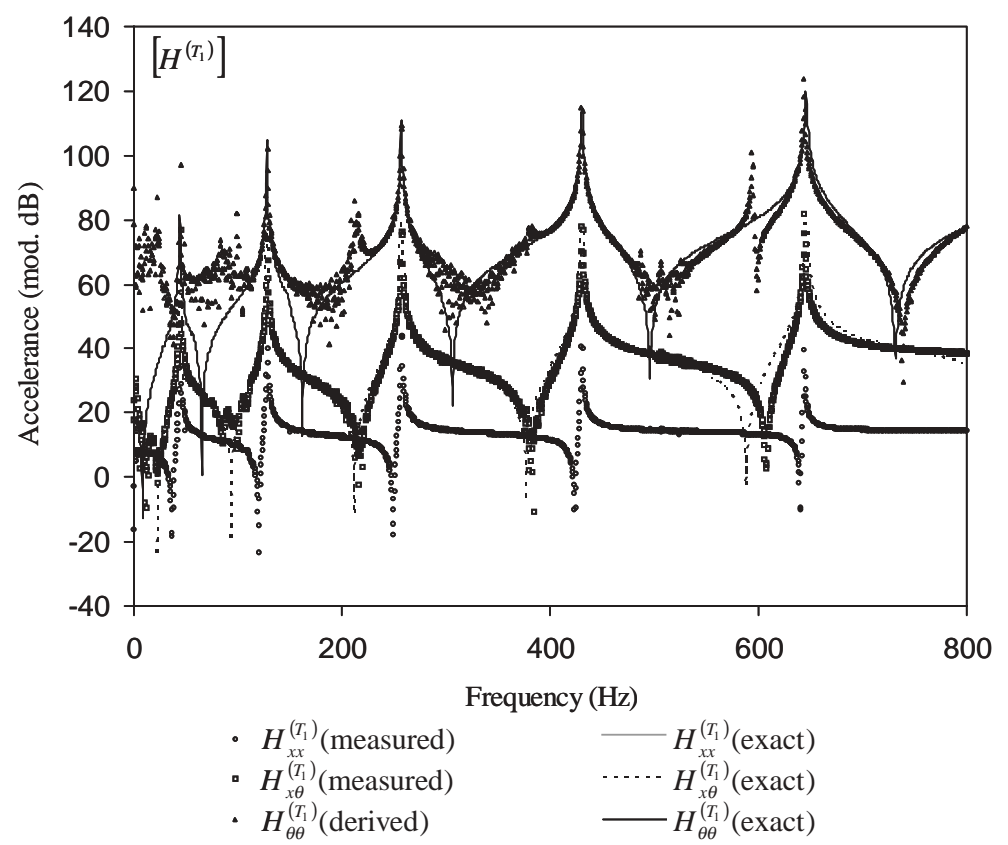

Fig. 13. Theoretical and Experimental terms of the accelerance matrix for system ' $T$ ' considering two co-ordinates $x$ and $\theta$.

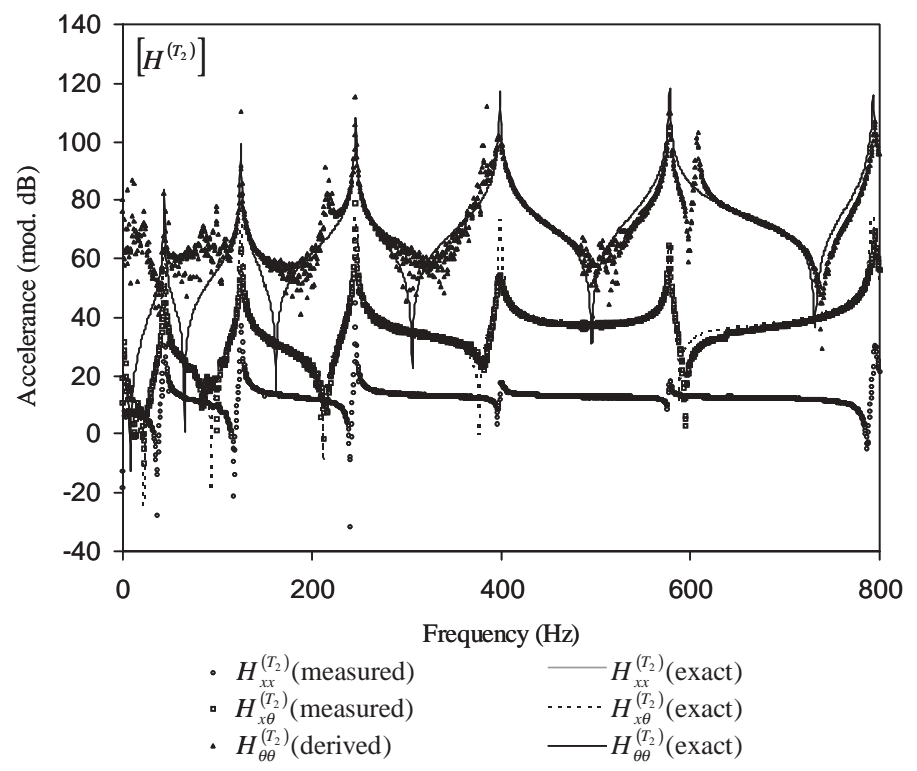

Fig. 14. Theoretical and Experimental terms of the accelerance matrix for system ' $T_{2}$ ' considering two co-ordinates $x$ and $\theta$.

where $\alpha_{j k}$ is the receptance relating the complex amplitude $\bar{X}_{j}$ of the dynamic response at co-ordinate $j$ to the amplitude $F_{i}$ of the exciting force at co-ordinate $i, \omega_{r}$ and $\eta_{r}$ are, respectively, the resonance frequency and hysteretic damping for mode $r,{ }_{r} \bar{A}_{j k}$ is a complex quantity known as Modal Constant and $N$ is the total number of modes.

Figures 15 and 16 summarize the procedure presenting the identified FRF curves and the theoretical ones superimposed so that the performance of the method can again be observed.

It is clear that the identification of the modal parameters eliminated random errors such as noise. However, its use was not good enough to minimize some spurious resonances and to bring out some anti-resonances that have 


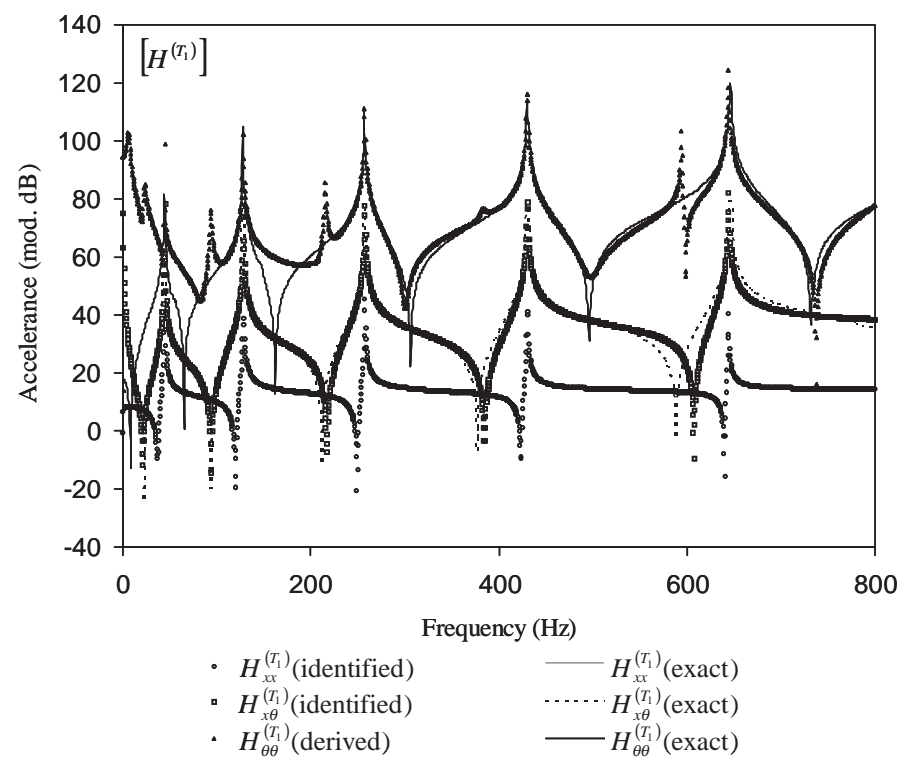

Fig. 15. Theoretical and Identified terms of the accelerance matrix for system ' $T_{1}$ ' considering two co-ordinates $x$ and $\theta$.

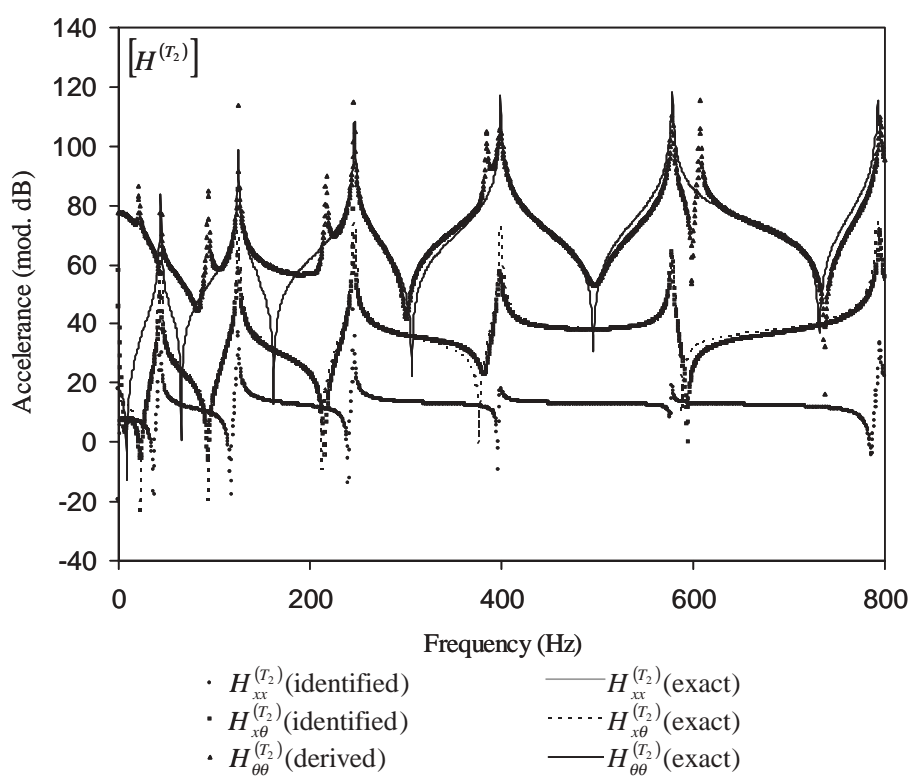

Fig. 16. Theoretical and Identified terms of the accelerance matrix for system ' $T_{2}$ ' considering two co-ordinates $x$ and $\theta$.

disappeared, which, according to the theoretical model and to the qualitative appreciation of curves $H_{x \theta}^{\left(T_{1}\right)}, H_{x \theta}^{\left(T_{1}\right)}$, $H_{x \theta}^{\left(T_{2}\right)}$ and $H_{x \theta}^{\left(T_{2}\right)}$, were to predict.

Since the determination of $H_{\theta \theta}^{\left(T_{1}\right)}$ and $H_{\theta \theta}^{\left(T_{2}\right)}$ depends exclusively on $H_{x \theta}^{\left(T_{1}\right)}$ and $H_{x \theta}^{\left(T_{2}\right)}$, it can be expected that the appearance of spurious resonances and the disappearance of anti-resonances are directly related to the experimental determination of $H_{x \theta}^{\left(T_{1}\right)}$ and $H_{x \theta}^{\left(T_{2}\right)}$. In fact, it can be seen that, in general, the spurious resonances of $H_{\theta \theta}^{\left(T_{1}\right)}$ are at the same frequency as the anti-resonances of $H_{x \theta}^{\left(T_{2}\right)}$. Also, under $300 \mathrm{~Hz}$ it is evident that some anti-resonances of $H_{\theta \theta}^{\left(T_{1}\right)}$ are missing. In that region, $H_{x \theta}^{\left(T_{1}\right)}$ and $H_{x \theta}^{\left(T_{2}\right)}$ are very similar and the numerical problem of subtractive cancellation 


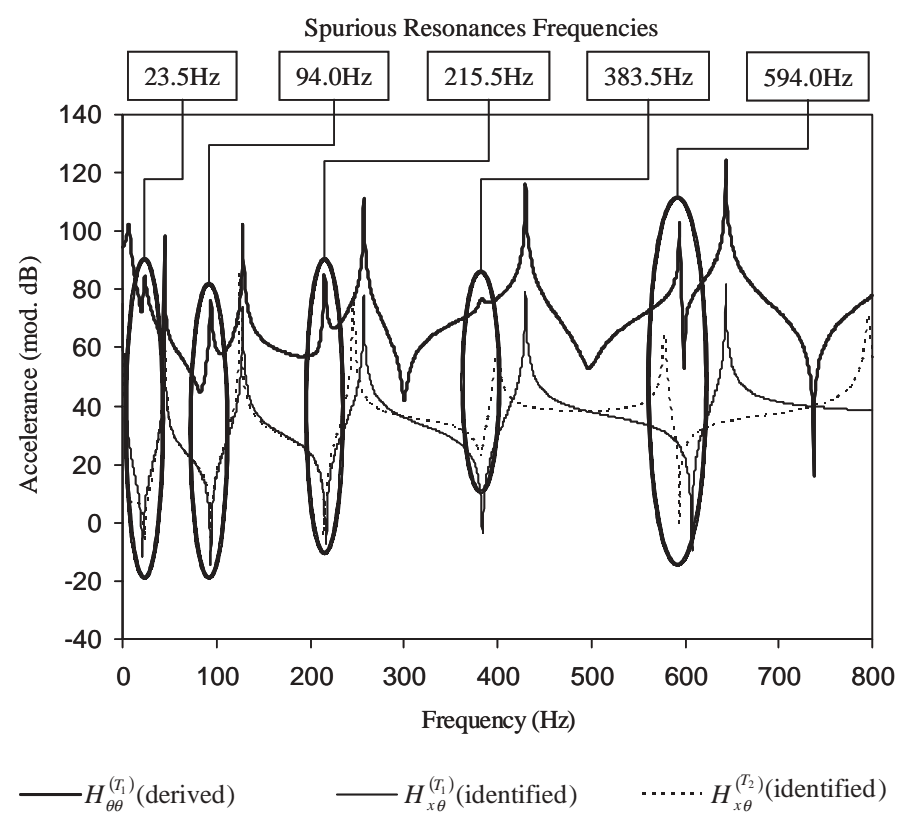

Fig. 17. Identified FRFs involved in Eq. (10). Analysis of possible causes to spurious resonances formation.

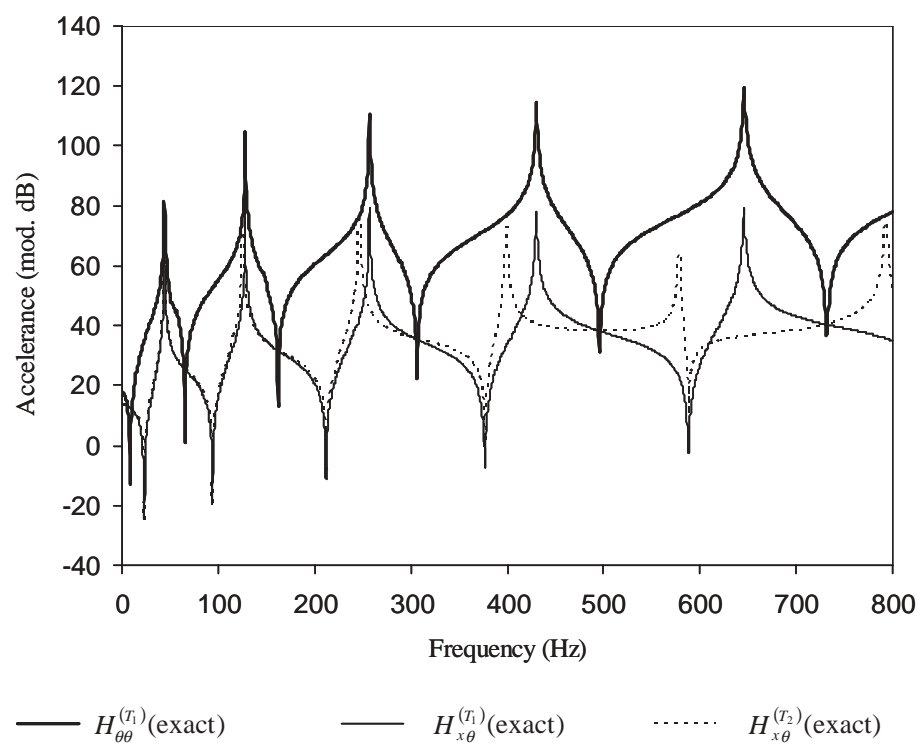

Fig. 18. Theoretical FRFs involved in Eq. (10) for comparison with Fig. 17.

arises. Furthermore, in that region, the method reveals to be more sensitive to identification of the modal parameters.

Figures 17 and 18 show, respectively, the theoretical and experimental (identified) $H_{\theta \theta}^{\left(T_{1}\right)}, H_{x \theta}^{\left(T_{1}\right)}$ and $H_{x \theta}^{\left(t_{2}\right)}$ curves superimposed for further discussion.

With attention to Fig. 17, almost all of the spurious resonances are at the same frequencies as the anti-resonances of $H_{x \theta}^{\left(T_{2}\right)}\left(H_{x \theta}^{\left(T_{2}\right)}\right.$ has an anti-resonance at $382 \mathrm{~Hz}$ which does not match the spurious resonance of $H_{\theta \theta}^{\left(T_{1}\right)}$ at $\left.383.5 \mathrm{~Hz}\right)$. This simple observation can be numerically justified if one remembers that expression (10), used to estimate $H_{\theta \theta}^{\left(T_{1}\right)}$, involves a quotient of $H_{x \theta}^{\left(T_{1}\right)}$ by $H_{x \theta}^{\left(T_{2}\right)}$. When $H_{x \theta}^{\left(T_{2}\right)}$ has a very small value, and if $H_{x \theta}^{\left(T_{1}\right)}$ has a much greater value 


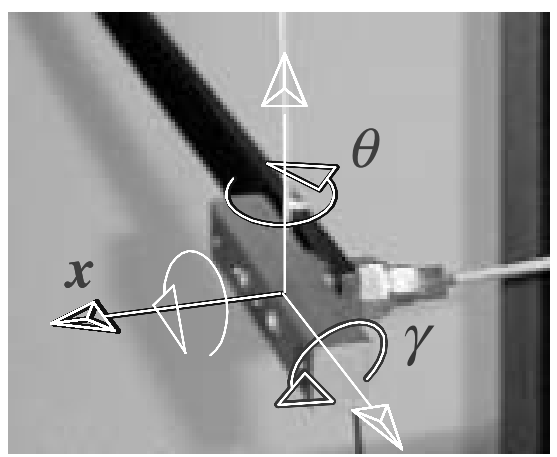

Fig. 19. New system of co-ordinates.

than $H_{x \theta}^{\left(T_{2}\right)}$, it is expected that this quotient has itself a big value. However, this problem does not occur in the theoretical model, as it can be seen by observation of Fig. 18, despite the fact that $H_{x \theta}^{\left(T_{2}\right)}$ has also very small values (when an anti-resonance is present). By comparing Fig. 17 to Fig. 18, one may notice that in the theoretical model the anti-resonances of $H_{x \theta}^{\left(T_{1}\right)}$ and $H_{x \theta}^{\left(T_{2}\right)}$ have coincident frequencies, though in the experimental model that is not observed. This is much more evident at higher frequencies, namely between $580 \mathrm{~Hz}$ and $620 \mathrm{~Hz}$.

Theoretically, it is known $[3,9,28]$ that a mass or rotational inertia modification at a given co-ordinate implies changes in the anti-resonances of all the FRFs that are not related to that co-ordinate and that the anti-resonances of those FRFs which are related to that specific co-ordinate are kept unchanged. Thus, with reference to the experimental model studied, a modification on the rotational inertia in co-ordinate $\theta$ should not shift the anti-resonance frequencies of curves $H_{x \theta}^{\left(T_{1}\right)}$ and $H_{x \theta}^{\left(T_{2}\right)}$. This is observed in Fig. 18, where a theoretical model is used, but not in Fig. 17, when an experimental model is present.

When exciting the beam, it is reasonable to believe that the force is not being applied in its neutral axis. In fact, it's very difficult to guarantee that the coupling point between the beam and the force transducer is exactly on the neutral axis, not only because of dimensional or geometric tolerances that have always to be considered, but also because we are dealing with a coupling area and not with a single coupling point. Besides other acceptable reasons, this seems to be the one that is more prominent in this case. This means that torsion is being excited, or at least, a rigid rotation towards the neutral fibber of the beam. Hence, though theoretically not considered, a rotational co-ordinate $\gamma$ is influencing the dynamic behaviour of the system with a relevant importance, as shown schematically in Fig. 19.

While rotating the T-block, it was considered that the only rotational inertia that was being changed was the one related to co-ordinate $\theta$. Therefore, it was expected that the anti-resonances of $H_{x \theta}^{\left(T_{1}\right)}$ and $H_{x \theta}^{\left(T_{2}\right)}$ would not exhibit shifts. However, changes in the rotational inertia towards co-ordinate $\theta$ also implies changes in the rotational inertia towards co-ordinate $\gamma$, and, accordingly, $H_{x \theta}^{\left(T_{1}\right)}$ and $H_{x \theta}^{\left(T_{2}\right)}$ are not expected to have anti-resonances at the same frequencies. The generic FRF matrix should now have the following aspect:

$$
[H]=\left[\begin{array}{lll}
H_{x x} & H_{x \theta} & H_{x \gamma} \\
H_{\theta x} & H_{\theta \theta} & H_{\theta \gamma} \\
H_{\gamma x} & H_{\gamma \theta} & H_{\gamma \gamma}
\end{array}\right]
$$

in which $H_{\gamma \theta}=H_{\theta \gamma}$ should be zero, because, in a beam, torsion and flexion modes are uncoupled.

In order to reinforce the idea that the anti-resonances shifting of $H_{x \theta}^{\left(T_{1}\right)}$ and $H_{x \theta}^{\left(T_{2}\right)}$ is a probable and reliable cause for the formation of spurious resonances, the anti-resonances of $H_{x \theta}^{\left(T_{2}\right)}$ were forced to match (in frequency) those belonging to $H_{x \theta}^{\left(T_{1}\right)}$. For this, it was used a procedure, based on the CRF modal identification technique, where the residual mass and stiffness [9] were determined so that the anti-resonances of $H_{x \theta}^{\left(T_{2}\right)}$ were shifted, in certain intervals, towards the anti-resonances of $H_{x \theta}^{\left(T_{1}\right)}$. Since it is not possible, for real structures, to measure all the modes $(N=\infty)$, the receptance given by Eq. (12) may be re-written as:

$$
\alpha_{j k}(\omega) \cong-\frac{1}{\omega^{2} \cdot M_{j k}^{R}}+\sum_{r=m_{1}}^{m_{2}}\left(\frac{{ }^{2} \bar{A}_{j k}}{\omega_{r}^{2}-\omega^{2}+i \eta_{r} \omega_{r}^{2}}\right)+\frac{1}{K_{j k}^{R}}
$$




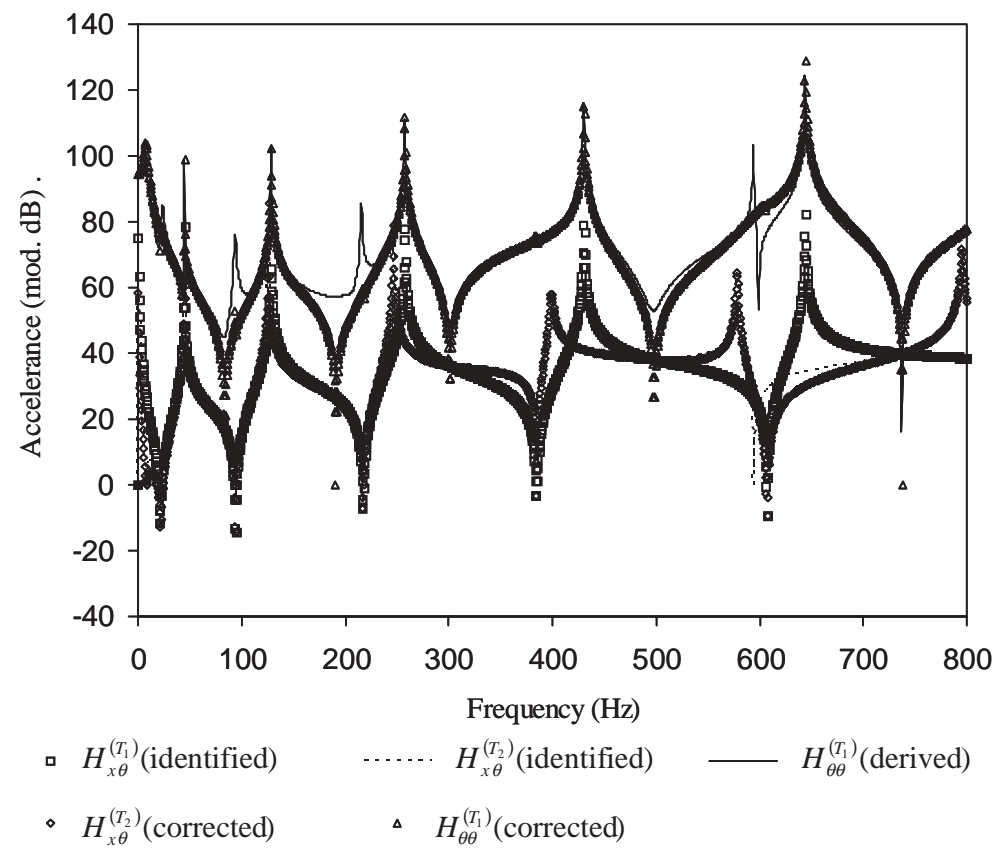

Fig. 20. Regeneration of the rotational accelerance of system ' $T_{1}$ ' by use of th residual mass and stiffness.

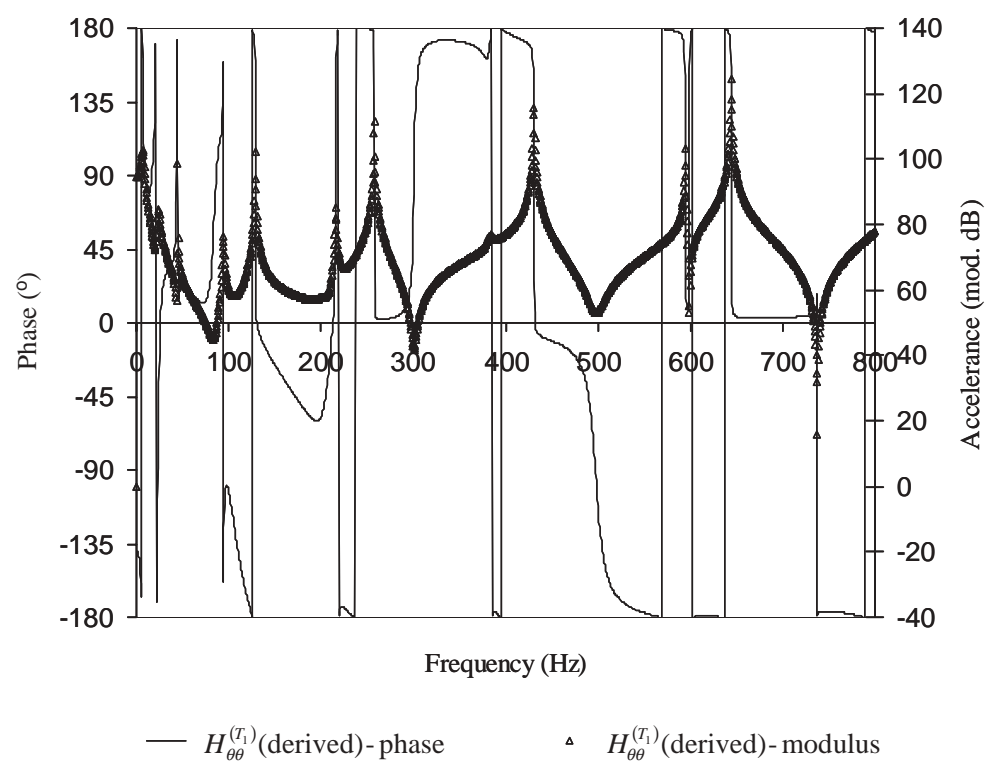

Fig. 21. Modulus and phase of the rotational accelerance of system ' $T_{1}$ ' obtained after identification of measured data.

in which $M_{j k}^{R}$ are $K_{j k}^{R}$, respectively, the residual mass and stiffness (complex) and $m_{1}$ and $m_{2}$ are, respectively, the lower and the higher limits of the frequency range of interest (the residuals are quantities that account for the influence of modes which are outside the frequency range).

Furthermore, it was imposed that $H_{\theta \theta}^{\left(T_{1}\right)}$ would have anti-resonances at its local minima. The results are shown in Fig. 20.

Though the results obtained by the correction procedure used are, apparently, very encouraging, one must not 

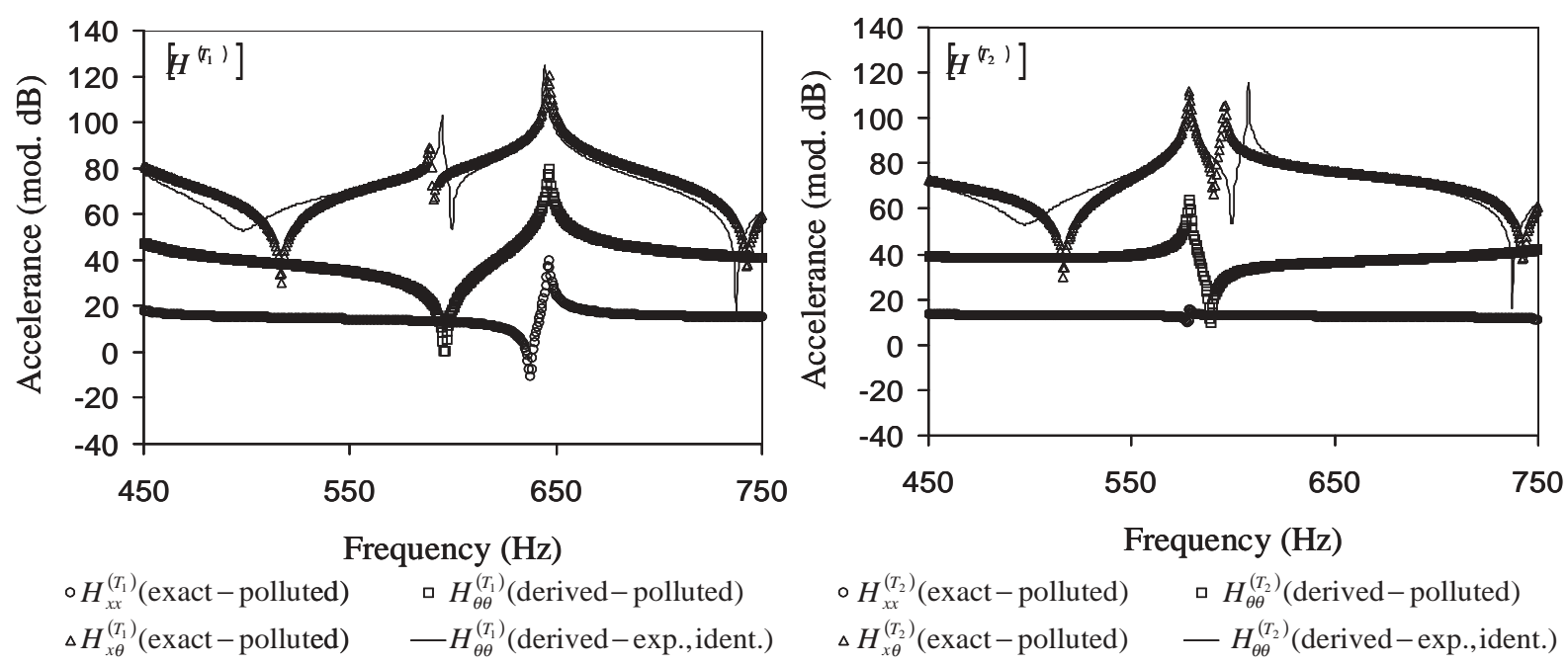

Fig. 22. Simulation of the influence of a rotation or torsion mode at co-ordinate $\gamma$ on theoretical results.

forget that the anti-resonances of the corrected $H_{\theta \theta}^{\left(T_{1}\right)}$ cannot be used as representative of the real dynamic behaviour of the structure, which is much more obvious for frequencies under $250 \mathrm{~Hz}$. This is a consequence of the method used in the determination of $H_{\theta \theta}^{\left(T_{1}\right)}$ anti-resonances, i.e., based on its local minima which may or may not correspond to anti-resonances. However, it has been shown that the formation of spurious resonances is most likely related to the "unexpected" shifting of the anti-resonances of curves $H_{x \theta}^{\left(T_{1}\right)}$ and $H_{x \theta}^{\left(T_{2}\right)}$.

Considering the above discussion, there is another question that must be more clearly understood, which is to evaluate whether the goal of trying to eliminate the spurious resonances is not in fact a way of eliminating the influence of torsion or rotation modes in co-ordinate $\gamma$. As a first approach, we shall analyse the phase of $H_{\theta \theta}^{\left(T_{1}\right)}$ superimposed to its modulus, as represented in Fig. 21.

Until $200 \mathrm{~Hz}$, it is very difficult to make any judgement concerning the relation between phase shifts and the modulus of $H_{\theta \theta}^{\left(T_{1}\right)}$. Still, above that frequency, general appreciation of the phase seems to support the possibility that the spurious resonances may in fact be resonances of torsion or rotation modes towards co-ordinate $\gamma$.

Finally, it was tried to simulate the influence of a rotation towards co-ordinate $\gamma$ starting with the theoretical curves. Considering that a rotation is occurring, the displacement measured by the response transducers is given by:

$$
x^{\prime}=x+\gamma \cdot d
$$

where $x$ is the translational displacement (which is the displacement considered in the theoretical model), $\gamma$ is the rotational displacement and $d$ is the arm distance. Thus, let us now consider, in the theoretical model, that $x_{A}=1.1 x_{A}$ for structure ' $T_{1}$ ' and let the other responses remain unchanged. For a matter of simplicity, let us also reduce the frequency range to $450 \mathrm{~Hz}$ to $750 \mathrm{~Hz}$, in order to cover the 5 th vibration mode. Figure 22 shows the obtained results.

Qualitatively, $H_{x \theta}^{\left(T_{1}\right)}, H_{x \theta}^{\left(T_{1}\right)}, H_{x \theta}^{\left(T_{2}\right)}$ and $H_{x \theta}^{\left(T_{2}\right)}$ curves seem unchanged when compared to the exact curves shown in Figs 15 and 16. However, the spurious resonances that appear in the derived curves $H_{\theta \theta}^{\left(T_{1}\right)}$ and $H_{\theta \theta}^{\left(T_{2}\right)}$ from theoretically polluted data are very similar in aspect to the derived curves and from identified experimental data. In fact, the result of amplification of the signal of structure ' $T_{1}$ ' in co-ordinate $x_{A}$ is a shift in the anti-resonance of $H_{x \theta}^{\left(T_{1}\right)}$, producing the formation of spurious resonances, which reinforces the idea that a rotation or a torsion mode is present in the analysis, with an impact that is of great importance.

\section{Conclusions}

A method to estimate rotational receptances without the need of generating a moment, based on coupling techniques, was presented and discussed in the numerical and experimental point of view. A rigid T-shaped block was 
used to include a rotational inertia modification by rotating it $90^{\circ}$ around one of its axis of symmetry. Translational responses were measured without physical contact by means of a two channel LASER vibrometer, resulting in rotational responses by subtraction. It has been shown that it is possible to obtain rotational FRFs without being necessary to apply excitation moments to the system under analysis. Cancellation of mass and rotational inertia effects can be easily done once the full receptance matrix at a point is known.

In summary, one can point out the following conclusions:

- When near a peek of vibration, where there is little rotation, the problem is ill-conditioned, as small changes in data generate big changes in results;

- Also, at low frequencies, estimation of rotational terms of the receptance matrix is often poor, as the FRFs involved in its derivation are very close in value;

- The identification of the modal parameters and regeneration of the experimental curves seem to eliminate problems related to random errors, such as noise. Nevertheless, when measuring near a peek of vibration or at low frequencies, the regeneration of the curves may introduce several changes on results if the modal identification process has not been carefully done;

- The appearance of spurious resonances in the rotational receptance $H_{\theta \theta}^{\left(T_{1}\right)}$, which is not avoided by the use of modal identification, is due to the "unexpected" relative shifts between the anti-resonances of the FRFs used in its derivation $\left(H_{x \theta}^{\left(T_{1}\right)}\right.$ and $\left.H_{x \theta}^{\left(T_{2}\right)}\right)$. In general, spurious resonances of $H_{\theta \theta}^{\left(T_{1}\right)}$ and anti-resonances of $H_{x \theta}^{\left(T_{2}\right)}$ are at the same frequency, reinforcing the previous statement.

- The anti-resonances shifting between $H_{x \theta}^{\left(T_{1}\right)}$ and $H_{x \theta}^{\left(T_{2}\right)}$ is a consequence of rotating the T-block, as a rotational displacement or a torsion mode may be present in a co-ordinate $\gamma$. In fact, by rotating the T-block, the rotaional inertia is being changed on both $\theta$ and $\gamma$ co-ordinates;

- The use of residual mass and stiffness to correct the rotational receptances showed that anti-resonances repositioning of $H_{x \theta}^{\left(T_{2}\right)}$ practically eliminates the spurious resonances of $H_{\theta \theta}^{\left(T_{1}\right)}$, though it was not possible to predict accurately the $H_{\theta \theta}^{\left(T_{1}\right)}$ anti-resonances.

\section{References}

[1] A.B. Stanbridge and D.J. Ewins, Using Continuous-Scan LDV Data for FE Model Validation in the Presence of 'Close' Modes, International Conference on Structural Dynamics Modelling, Funchal, Madeira, Portugal, 2002, pp. 267-276.

[2] A.M.R. Ribeiro, Development of Dynamic Analysis Technics for Structural Modification, Ph.D. Dissertation, Instituto Superior Ecnico, Technical University of Lisbon, Portugal, 1999, in Portuguese.

[3] D. Afolabi, An Anti-Resonance Technique for Detecting Structural Damage, Proc. of the 5th International Modal Analysis Conference (IMAC V), London, United Kingdom, 1987, pp. 491-495.

[4] D.H. Soyster and M.W. Trethewey, Use of Rotational Elements in Structural Dynamic Modification, The International Journal of Analytical and Experimental Modal Analysis 11(1) (1996), 76-82.

[5] D.J. Ewins and M.G. Sainsbury, Mobility Measurements for the Vibration Analysis of Connected Structures, The Shock and Vibration Bulletin 42(1) (1972), 105-122.

[6] D.J. Ewins and P.T. Gleeson, Experimental Determination of Multi Directional Mobility Data for Beams, The Shock and Vibration Bulletin 45(5) (1975), 153-173.

[7] D.J. Ewins and J.M.M. Silva, Measurements of Structural Mobility on Helicopter Structures, Symposium on Internal Noise in Helicopters, University of Southampton, United Kingdom, 1979.

[8] D.J. Ewins, Modal Testing: Theory and Practice, 2nd ed., Research Studies Press Ltd, 1984.

[9] D. Montalvão, Determination of Rotational Terms of the Dynamic Response by means of Modal Analysis Techniques, M.Sc. Dissertation, Instituto Superior Técnico, Technical University of Lisbon, Portugal, 2003, (in Portuguese).

[10] E.H. Bokelberg, H.J. Sommer III and M.W. Trethewey, A Six-Degree-of-Freedom Laser Vibrometer, Part I: Theoretical Development, Journal of Sound and Vibration 178(5) (1994), 643-654.

[11] E.H. Bokelberg, H.J. Sommer III and M.W. Trethewey, A Six-Degree-of-Freedom Laser Vibrometer, Part II: Experimental Validation, Journal of Sound and Vibration 178(5) (1994), 655-667.

[12] E.J. Smith, Measurement of the Total Structural Mobility Matrix, The Shock and Vibration Bulletin 40(7) (1969), 51-84.

[13] EU Contract BRPR-CT97-0540, Project number BE 97-4184, Quantitative Treatment and Testing of Rotational Degrees of Freedom (QUATTRO): Guidelines for experimental techniques, www.dem.ist.utl.pt/ ${ }^{2}$ QUATTRO/, 2000.

[14] G.R. Cowper, The Shear Coefficient in Timoshenko's Beam Theory, Journal of Applied Mechanics 335 (1966).

[15] H. Pina, Métodos Numéricos, McGraw-Hill, 1995, in Portuguese.

[16] J.A. Cafeo, M.W. Trethewey and H.J. Sommer, Measurement and Application of Experimental Rotational Degrees of Freedom for Mode Shape Refinement, The International Journal of Analytical and Experimental Modal Analysis 7(4) (1992), $255-269$. 
[17] J.E. Mottershead, A. Kyprianou and H. Ouyang, Estimation of Rotational Frequency Responses, Proc. of the 5th International Conference on Damage Assessment of Structures (DAMAS 2003), Transtech Publications, Southampton, United Kingdom, 2003 , pp. 157-165.

[18] J.E. Mottershead, A. Kyprianou and H. Ouyang, Structural Modification, Part 1: Rotational Receptances, 2003, to appear.

[19] J.M.M. Silva, The Influence of the Joint on the Vibration Response of a Cross-Beam Assembly, M.Sc. Dissertation, Imperial College of Science and Technology, University of London, United Kingdom, 1974.

[20] J.M.M. Silva, Measurements and Applications of Structural Mobility Data for the Vibration Analysis of Complex Structures, Ph.D. Dissertation, Imperial College of Science and Technology, University of London, United Kingdom, 1978.

[21] J.M.M. Silva, N.M.M. Maia and A.M.R. Ribeiro, Estimation of Frequency Response Functions Involving Rotational DOF's using an Uncoupling Technique, Proc. of the International Conference on Applications of Modal Analysis, Gold Coast, Queensland, Australia, 1999, pp. 15-17.

[22] J.M.M. Silva, N.M.M. Maia and A.M.R. Ribeiro, Cancellation of Mass-Loading Effects of Transducers and Evaluation of Unmeasured Frequency Response Functions, Journal of Sound and Vibration 236(5) (2000), 761-779.

[23] M.A. Sanderson and C.R. Fredo, Direct Measurement of Moment Mobility, Part I: A Theoretical Study, Journal of Sound and Vibration 179(4) (1995), 669-684.

[24] M.A. Sanderson, Direct Measurement of Moment Mobility, Part II: An Experimental Study, Journal of Sound and Vibration 179(4) (1995), 685-696.

[25] M.G. Sainsbury, Experimental and Theoretical Techniques for the Vibration Analysis of Damped Complex Structures, Ph.D. Dissertation, Imperial College of Science and Technology, University of London, United Kingdom, 1976.

[26] M.W. Trethewey and H.J. Sommer III, Measurement of Rotational DOF Frequency Response Functions with Pure Moment Excitation, International Conference on Structural Dynamics Modelling, Funchal, Madeira, Portugal, 2002, pp. 81-88.

[27] N.M.M. Maia, A.M.R. Ribeiro and J.M.M. Silva, A New Concept in Modal Analysis: the Characteristic Response Function, International Journal of Analytical and Experimental Modal Analysis 9(3) (1994), 191-202.

[28] N.M.M. Maia and J.M.M. Silva, Theoretical and Experimental Modal Analysis, 1st ed., Research Studies Press Ltd., 1997.

[29] N.M.M. Maia, J.M.M. Silva and A.M.R. Ribeiro, Some Applications of Coupling/Uncoupling Techniques in structural Dynamics - Part 2: Generation of the Whole FRF Matrix from Measurements on a Single Column - The Mass Uncoupling Method, Proc. of the 15th International Modal Analysis Conference (IMAC XV), Orlando, Florida, USA, 1997.

[30] N.M.M. Maia, J.M.M. Silva and A.M.R. Ribeiro, Some Applications of Coupling/Uncoupling Techniques in structural Dynamics - Part 3: Estimation of Rotational Frequency-Response-Functions using MUM, Proc. of the 15th International Modal Analysis Conference (IMAC XV), Orlando, Florida, USA, 1997.

[31] P.T. Gleeson, Identification of Spatial Models for the Vibration Analysis of Lightly Damped Structures, Ph.D. Dissertation, Imperial College of Science and Technology, University of London, United Kingdom, 1979.

[32] W. D'Ambrogio and A. Sestieri, Substructure Coupling using FRFs: Strategies for Tackling Rotational Degrees of Freedom, Proc. of the International Conference on Structural Dynamics Modelling, Funchal, Madeira, Portugal, 2002, pp. 229-238. 

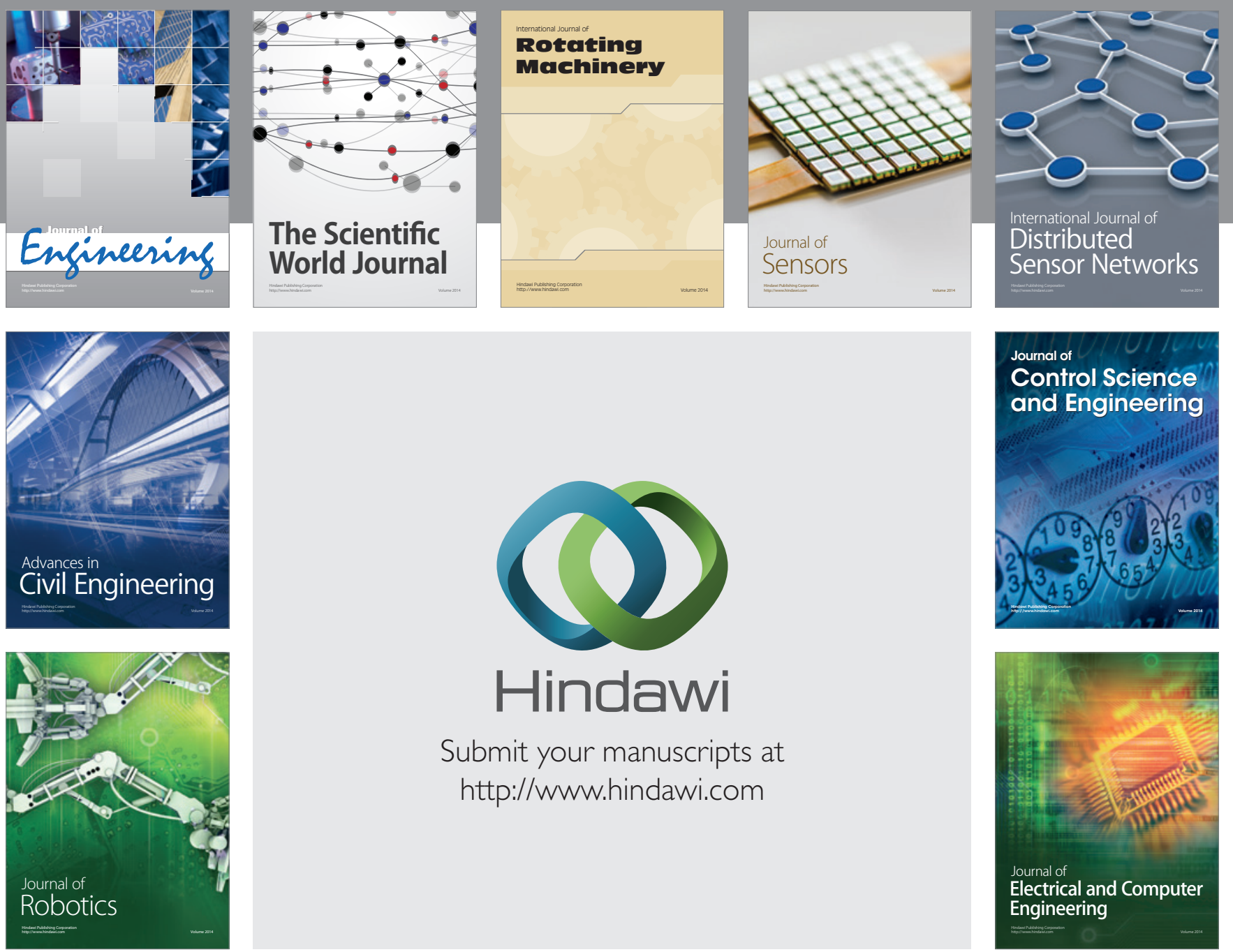

Submit your manuscripts at

http://www.hindawi.com
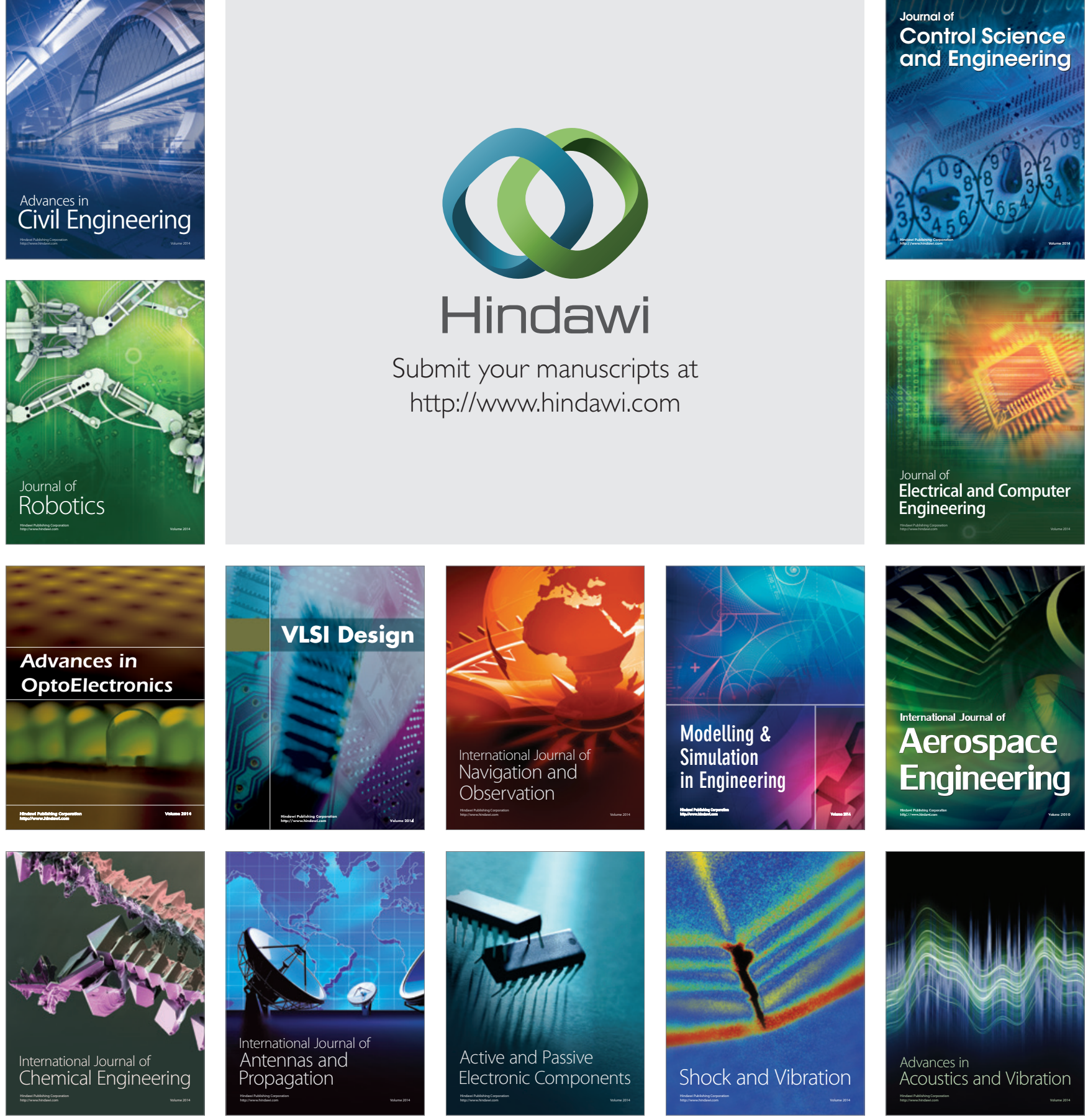